\title{
French Translation of the Perth Alexithymia Questionnaire
}

\author{
Olivier Luminet \\ UCLouvain, Belgium \\ olivier.luminet@uclouvain.be \\ Carole Fantini-Hauwel \\ Université Libre de Bruxelles, Belgium \\ carole.fantini@ulb.be \\ Emilie Banse \\ UCLouvain, Belgium \\ emilie.banse@student.uclouvain.be \\ Alix Bigot \\ UCLouvain, Belgium \\ alix.bigot@student.uclouvain.be \\ David A. Preece \\ Curtin University \& University of Western Australia, Australia \\ david.preece@curtin.edu.au
}

\section{Processus de traduction}

Cette traduction française du Perth Alexithymia Questionnaire $(\mathrm{PAQ})$ a été réalisée à partir de la version originale anglaise. Une première traduction de l'anglais vers le français a été réalisée et affinée par l'équipe des auteurs. Ces items français ont ensuite été retraduits en anglais par un traducteur agréé par le NAATI. Des améliorations mineures ont été apportées à certains items français par l'équipe des auteurs sur la base de cette retraduction, pour aboutir à la version française finale du PAQ présentée dans ce document.

Notre équipe mène actuellement des études psychométriques sur la version française du PAQ afin d'en examiner la validité et la fiabilité.

\section{Translation process}

This French translation of the Perth Alexithymia Questionnaire (PAQ) was translated from the original English version. An initial translation from English to French was conducted and refined by the authorship team. These French items were then back-translated into English by a NAATI approved translator. Minor refinements to some of the French items were made by the authorship team on the basis of this back-translation, resulting in the final French version of the PAQ presented in this document.

Our team is currently conducting a psychometric study on this French PAQ to examine its validity and reliability. 
Ce questionnaire porte sur la façon dont vous percevez et ressentez vos émotions. Veuillez indiquer, pour les affirmations suivantes, dans quelle mesure vous êtes d'accord ou non avec le fait que cette proposition s'applique à vous (ou est vraie pour vous). Encerclez une réponse pour chaque affirmation.

Certaines questions mentionnent des émotions négatives ou désagréables, comme la tristesse, la colère, ou la peur. Certaines questions mentionnent des émotions positives ou agréables, comme la joie, l'amusement ou l'excitation.

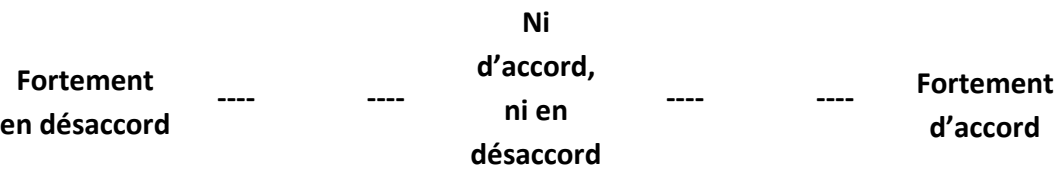

Quand je ne me sens pas bien (ressens une émotion désagréable), je n'arrive pas à trouver les bons mots pour décrire ces

$\begin{array}{lllllll}1 & 2 & 3 & 4 & 5 & 6 & 7\end{array}$
sentiments.

Quand je ne me sens pas bien, je ne sais pas si je suis triste, effrayé(e) ou en colère.

$\begin{array}{lllllll}1 & 2 & 3 & 4 & 5 & 6 & 7\end{array}$

J'ai tendance à ne pas faire attention à ce que ressens.

1

Quand je me sens bien (ressens une

4 émotion agréable), je n'arrive pas à trouver les bons mots pour décrire ces sentiments.

Quand je me sens bien, je ne sais pas si je suis heureux(se), excité(e) ou amusé(e).

1

1

2

1 plan plutôt que de me concentrer sur cellesci.

Quand je ne me sens pas bien, je n'arrive pas à parler de mes émotions en 1 profondeur ou en détails.

Quand je ne me sens pas bien, je n'arrive pas à donner un sens à mes émotions.

1

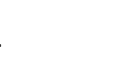

1

1

1

donner un sens à mes émotions. 


\begin{tabular}{|c|c|c|c|c|c|c|c|c|}
\hline & & $\begin{array}{l}\text { Fortement } \\
\text { en désaccord }\end{array}$ & ---- & --- & $\begin{array}{l}\mathrm{Ni} \\
\text { d'accord, } \\
\text { ni en } \\
\text { désaccord }\end{array}$ & ---- & ---- & $\begin{array}{l}\text { Fortement } \\
\text { d'accord }\end{array}$ \\
\hline 13 & $\begin{array}{l}\text { Quand quelque chose de négatif se produit, } \\
\text { il est difficile pour moi de mettre des mots } \\
\text { sur ce que je ressens. }\end{array}$ & 1 & 2 & 3 & 4 & 5 & 6 & 7 \\
\hline 14 & $\begin{array}{l}\text { Quand je ne me sens pas bien, je suis } \\
\text { confus(e) par rapport à l'émotion que je } \\
\text { ressens. }\end{array}$ & 1 & 2 & 3 & 4 & 5 & 6 & 7 \\
\hline 15 & $\begin{array}{l}\text { Je préfère me concentrer sur les choses que } \\
\text { je peux vraiment voir ou toucher, plutôt } \\
\text { que sur mes émotions. }\end{array}$ & 1 & 2 & 3 & 4 & 5 & 6 & 7 \\
\hline 16 & $\begin{array}{l}\text { Quand quelque chose de bien se produit, il } \\
\text { est difficile pour moi de mettre des mots } \\
\text { sur ce que je ressens. }\end{array}$ & 1 & 2 & 3 & 4 & 5 & 6 & 7 \\
\hline 17 & $\begin{array}{l}\text { Quand je me sens bien, je suis confus(e) par } \\
\text { rapport à l'émotion que je ressens. }\end{array}$ & 1 & 2 & 3 & 4 & 5 & 6 & 7 \\
\hline 18 & $\begin{array}{l}\text { Je n'essaye pas d'être en contact avec mes } \\
\text { émotions. }\end{array}$ & 1 & 2 & 3 & 4 & 5 & 6 & 7 \\
\hline 19 & $\begin{array}{l}\text { Quand je ne me sens pas bien, si j'essaye de } \\
\text { décrire ce que je ressens, je ne sais pas quoi } \\
\text { dire. }\end{array}$ & 1 & 2 & 3 & 4 & 5 & 6 & 7 \\
\hline 20 & $\begin{array}{l}\text { Quand je ne me sens pas bien, je suis } \\
\text { désorienté par ce que je ressens. }\end{array}$ & 1 & 2 & 3 & 4 & 5 & 6 & 7 \\
\hline 21 & $\begin{array}{l}\text { Ce n'est pas important pour moi de savoir } \\
\text { ce que je ressens. }\end{array}$ & 1 & 2 & 3 & 4 & 5 & 6 & 7 \\
\hline 22 & $\begin{array}{l}\text { Quand je me sens bien, si j'essaye de } \\
\text { décrire ce que je ressens, je ne sais pas quoi } \\
\text { dire. }\end{array}$ & 1 & 2 & 3 & 4 & 5 & 6 & 7 \\
\hline 23 & $\begin{array}{l}\text { Quand je me sens bien, je suis désorienté } \\
\text { par ce que je ressens. }\end{array}$ & 1 & 2 & 3 & 4 & 5 & 6 & 7 \\
\hline 24 & $\begin{array}{l}\text { C'est bizarre pour moi de penser à mes } \\
\text { émotions. }\end{array}$ & 1 & 2 & 3 & 4 & 5 & 6 & 7 \\
\hline
\end{tabular}




\section{Perth Alexithymia Questionnaire (PAQ) Scoring Instructions}

L'alexithymie est un construit multidimensionnel qui comprend trois dimensions: les difficultés à identifier ses propres émotions (DIF), les difficultés à décrire et communiquer ses propres émotions (DDF) et le style de pensée orienté vers l'extérieur (EOT) par lequel les personnes tendent à ne pas se focaliser sur les émotions, à ne pas leur prêter attention. En d'autres termes, les personnes qui ont un niveau élevé d'alexithymie ont des difficultés à focaliser leur attention sur leurs états émotionnels (EOT), et des difficultés à évaluer correctement ce que sont ces états émotionnels (DIF, DDF) (Preece et al., 2017 ; Sifneos, 1973).

Le questionnaire PAQ (Preece et al., 2018) est une mesure d'alexithymie auto-rapportée en 24 items. Il est construit pour mesurer toutes les composantes de l'alexithymie et ce, pour les émotions négatives ET positives. Cinq scores de sous-échelles et six scores composites peuvent être obtenus à partir du questionnaire, les scores élevés indiquant de plus hauts niveau d'alexithymie. Pour plus d'informations à propos de la validité et de la fiabilité du PAQ, voir Preece et al. (2018, 2020a, 2020b, 2020c) ainsi que Greene et al. (2020).

Le tableau ci-dessous décrit chacune des sous échelles et des scores composites du PAQ ainsi que la manière de les calculer. Nous avons également créé une feuille de calcul Excel qui calcule les scores automatiquement. Cette feuille de scoring peut être téléchargée ici : https://www.researchgate.net/publication/329058432. Elle inclut également des données normatives pour vous aider à interpréter les scores du PAQ. Nous souhaitons continuer à mettre à jour cette feuille de scoring à mesure que de nouvelles données normatives deviennent disponibles.

\begin{tabular}{|c|c|c|}
\hline \multicolumn{3}{|c|}{ Comment calculer? } \\
\hline \multirow{2}{*}{\multicolumn{3}{|c|}{\begin{tabular}{l|l}
\multicolumn{2}{c}{ scores aux sous-échelles } \\
1,20 & Difficultés
\end{tabular}}} \\
\hline & & \\
\hline DIfficultés à identifier les émotions-Positives (P-DIF) & Somme des items 5, 11, 17, 23 & Difficultés à identifier, comprendre et différencier ses propres émotions positives \\
\hline DIfficultés à décrire les émotions- Négatives (N-DDF) & Somme des items 1, 7, 13, 19 & Difficultés à décrire et communiquer ses propres émotions négatives \\
\hline DIfficultés à décrire les émotions- Positives (P-DDF) & Somme des items $4,10,16,22$ & Difficultés à décrire et communiquer ses propres émotions positives \\
\hline Pensée orientée vers l'extérieur-Général (G-EOT) & Somme des items $3,6,9,12,15,18,21,24$ & $\begin{array}{l}\text { Tendance à ne pas focaliser son attention sur ses propres émotions (négatives et } \\
\text { positives) }\end{array}$ \\
\hline \multicolumn{3}{|c|}{ scores composites } \\
\hline Difficultés à identifier les émotions-Général (G-DIF) & Somme des sous-échelles N-DIF et P-DIF & $\begin{array}{l}\text { Difficultés à identifier, comprendre et différencier ses propres émotions (négatives et } \\
\text { positives) }\end{array}$ \\
\hline Difficultés à décrire les émotions-Général (G-DDF) & Somme des sous-échelles N-DDF et P-DDF & Difficultés à décrire et communiquer ses propres émotions (négatives et positives) \\
\hline Difficultés à évaluer les émotions- Négatives (N-DAF) & Somme des sous-échelles N-DIF et N-DDF & Difficultés à identifier et décrire (i.e., évaluer) ses propres émotions négatives. \\
\hline Difficultés à évaluer les émotions- Positives (P-DAF) & Somme des sous-échelles P-DIF et P-DDF & Difficultés à identifier et décrire (i.e., évaluer) ses propres émotions positives \\
\hline Difficultés à évaluer les émotions- Général (G-DAF) & $\begin{array}{l}\text { Somme des sous-échelles N-DIF, P-DIF, N- } \\
\text { DDF, P-DDF }\end{array}$ & $\begin{array}{l}\text { Difficultés à identifier et décrire (i.e., évaluer) ses propres émotions (négatives et } \\
\text { positives) }\end{array}$ \\
\hline Alexithymie (Score total) & Somme de tous les items & $\begin{array}{l}\text { Alexithymie globale; difficultés à focaliser son attention et évaluer ses propres émotions } \\
\text { (négatives et positives) }\end{array}$ \\
\hline
\end{tabular}




\section{PAQ statistiques descriptives}

Les statistiques descriptives et les coefficients alpha de Cronbach issus d'un échantillon général d'adultes australiens ( $N=748$; Preece et al., 2018) sont présentés dans le tableau ci-dessous.

\begin{tabular}{|c|c|c|c|c|c|c|c|}
\hline \multirow[b]{2}{*}{$\begin{array}{l}\text { Sous-échelle:scores } \\
\text { composites }\end{array}$} & \multicolumn{3}{|c|}{ Echantillon total ( $N=748)$} & \multicolumn{2}{|c|}{ Femmes ( $N=468)$} & \multicolumn{2}{|c|}{ Hommes $(N=280)$} \\
\hline & $M$ & $S D$ & $\begin{array}{l}\text { Cronbach's } \\
\text { alpha }\end{array}$ & $M$ & $S D$ & $M$ & $S D$ \\
\hline \multicolumn{8}{|l|}{ Sous-échelles } \\
\hline $\mathrm{N}$-DIF & 13.38 & 6.41 & 0.89 & 14.03 & 6.57 & 12.28 & 5.98 \\
\hline P-DIF & 11.30 & 5.76 & 0.89 & 11.38 & 6.08 & 11.18 & 5.20 \\
\hline $\mathrm{N}-\mathrm{DDF}$ & 15.35 & 6.89 & 0.91 & 15.80 & 7.00 & 14.60 & 6.63 \\
\hline P-DDF & 12.97 & 6.12 & 0.90 & 12.72 & 6.24 & 13.38 & 5.90 \\
\hline G-EOT & 28.97 & 11.19 & 0.90 & 27.92 & 11.35 & 30.74 & 10.72 \\
\hline \multicolumn{8}{|l|}{ Composites } \\
\hline G-DIF & 23.68 & 11.28 & 0.92 & 25.41 & 11.57 & 23.45 & 10.68 \\
\hline G-DDF & 28.32 & 12.16 & 0.93 & 28.52 & 12.30 & 27.98 & 11.92 \\
\hline N-DAF & 28.73 & 12.71 & 0.94 & 29.83 & 13.07 & 26.88 & 11.89 \\
\hline P-DAF & 24.27 & 11.39 & 0.94 & 24.1 & 11.95 & 24.55 & 10.41 \\
\hline G-DAF & 52.99 & 22.58 & 0.96 & 53.93 & 23.23 & 51.43 & 21.39 \\
\hline Score total & 81.97 & 30.91 & 0.96 & 81.84 & 31.92 & 82.17 & 29.15 \\
\hline
\end{tabular}

\section{Interprétations des scores}

Pour interpréter les scores du PAQ, il est préférable que le score d'un répondant soit comparé aux scores issus d'un échantillon normatif adéquat. L'alexithymie est un construit dimensionnel (plutôt que catégoriel) qui est normalement distribué dans la population générale. Il est donc préférable de considérer les scores d'alexithymie le long d'un continuum. Chaque personne possède un certain niveau d'alexithymie, qu'il soit bas, modéré ou élevé. Le nombre de déviation standard (SD) du score d'un répondant autour de la moyenne issue d'un échantillon normatif adéquat renseigne sur le niveau d'alexithymie. Nous interprétons les scores du PAQ de la manière suivante, en gardant à l'esprit qu'un score élevé indique un niveau d'alexithymie élevé :

- Score $>1$ SD ou plus de la moyenne = « haut niveau d'alexithymie »

- Score $\leq 1$ SD de la moyenne = « niveau modéré d'alexithymie »

- Score < 1 SD ou plus de la moyenne = « faible niveau d'alexithymie » 


\section{References}

Greene, D., Hasking, P., Boyes, M., \& Preece, D. (2020). Measurement Invariance of Two Measures of Alexithymia in Students Who Do and Who Do Not Engage in Non-suicidal Self-Injury and Risky Drinking. Journal of Psychopathology and Behavioral Assessment, 1-18.

Preece, D., Becerra, R., Allan, A., Robinson, K., \& Dandy, J. (2017). Establishing the theoretical components of alexithymia via factor analysis: Introduction and validation of the attention-appraisal model of alexithymia. Personality and Individual Differences, 119, 341-352.

Preece, D., Becerra, R., Robinson, K., Dandy, J., \& Allan, A. (2018). The psychometric assessment of alexithymia: Development and validation of the Perth Alexithymia Questionnaire. Personality and Individual Differences, 132, 32-44.

Preece, D. A., Becerra, R., Boyes, M. E., Northcott, C., McGillivray, L., \& Hasking, P. A. (2020a). Do self-report measures of alexithymia measure alexithymia or general psychological distress? A factor analytic examination across five samples. Personality and Individual Differences, 155, 1-8.

Preece, D. A., Becerra, R., Allan, A., Robinson, K., Chen, W., Hasking, P., \& Gross, J. J. (2020b). Assessing alexithymia: Psychometric properties of the Perth Alexithymia Questionnaire and 20-item Toronto Alexithymia Scale in United States adults. Personality and Individual Differences, 166, 1-8.

Preece, D. A., Becerra, R., Robinson, K., Allan, A., Boyes, M., Chen, W., Hasking, P., \& Gross, J. J. (2020c). What is Alexithymia? Using Factor Analysis to Establish its Latent Structure and Relationship with Fantasizing and Emotional Reactivity. Journal of Personality, 1-15.

Sifneos, P. E. (1973). The prevalence of 'alexithymic' characteristics in psychosomatic patients. Psychotherapy and Psychosomatics, 22, 255-262. 